\title{
Two-dimensional Bloch electrons under strong magnetic modulation
}

\author{
J. L. Movilla and J. Planelles* \\ Departament de Química Física i Analítica, Universitat Jaume I, Box 224, E-12080 Castelló, Spain \\ (Received 9 September 2010; revised manuscript received 11 November 2010; published 13 January 2011)
}

\begin{abstract}
The band structure of a high-mobility two-dimensional electron gas patterned with a square lattice of holes (antidots) is studied theoretically under the influence of a magnetic modulation consisting of perpendicular magnetic flux tubes with the same period and nonzero net flux per unit cell. The magnetic field pierces the system through the patterned holes only, so that the coupling with the electrons is purely quantum mechanical. The model takes implicitly into account the coupling between the different Bloch bands. The flux-dependent energy structure exhibits a Hofstadter butterfly-type spectrum. Such a structure is repeated indefinitely without distortion with a period of one magnetic flux quantum through a lattice hole. Rectangular deviations from the square lattice are also studied. It is found that the number and width of the magnetic gaps decrease, and even disappear for large antidot filling fractions.
\end{abstract}

DOI: 10.1103/PhysRevB.83.014410

PACS number(s): 73.21.Cd, 73.20.At

\section{INTRODUCTION}

The preparation of well-defined periodic structures imposed onto the plane of the two-dimensional electron system (2DES) and its effects on the transport properties under applied magnetic fields have become the object of intense studies over the last two decades. ${ }^{1}$ The initial motivation for studying magnetotransport in such structures was the opportunity to access the regime of the Hofstadter butterfly energy spectrum. ${ }^{2}$ This spectrum, calculated initially in the one-band tightbinding approach, exhibits a highly aesthetic, self-similar structure of energy bands and gaps, whose number and widths are changing with the magnetic flux threading the unit cell of the 2D lattice. Many efforts have been devoted to the challenge of observing signatures of this fractal energy spectrum, mainly in lateral superlattices patterned on highmobility GaAs/AlGaAs heterostructures. ${ }^{4-7}$ A remnant of the nonmonotonic behavior of the Hall conductivity peculiar to the Hofstadter butterfly has been reported. ${ }^{6}$ However, for many years there has been no conclusive experimental evidence for this structure, presumably because the small gaps between subbands tend to close due to disorder in samples of not exceptionally high mobilities. ${ }^{8-10}$ Additionally, Hofstadter's high-symmetry picture has been shown to undergo dramatic distortions in conditions departing from the limiting case of very weak lattice potentials. ${ }^{1,3,4}$

Magnetotransport studies on square, ${ }^{7,11-14}$ rectangular, ${ }^{15-17}$ or hexagonal ${ }^{18,19}$ superlattices have revealed a variety of typical phenomena, such as different types of characteristic magnetoresistance oscillations. Features in the magnetoresistance are commonly ascribed to classical or semiclassical effects, though the discussion on the nature of the characteristic oscillations is still open. ${ }^{20}$ Even quantum mechanically, some of these features can be explained independently of the butterfly subband structure, focusing exclusively on the approximate band structure (i.e., on the characteristics of the envelope function that modulates the butterfly-type spectrum). ${ }^{21}$

The system we propose in this paper could be useful to provide indirectly further understanding on the features observed in the low-field magnetoresistance of electrically modulated 2DES, since in the proposed setup the magnetic field does not pierce the electron gas, so we eliminate the classical contribution of the magnetic field to the electron motion. As a result, the exhibited Hofstadter-type subband structure is not distorted by the magnetic field, thus yielding an unmodulated envelope; the band structure is repeated in the field axis indefinitely without distortion, with a period of one flux quantum piercing the lattice unit cell. This property could also turn it into a suitable system under the perspective of observing aspects of the butterfly spectrum, ${ }^{22}$ free of most of the distortions inherent in typically studied 2DES with electric or magnetic modulations., 3,23 The system proposed is shown schematically in Fig. 1. It consists of a high-mobility semiconductor heterostructure (such as GaAs/GaAlAs) with a superconducting layer grown on top of it. The whole system is patterned with a lattice of nanometer-sized holes, which act as forbidden regions (antidots) for the 2D electrons. A homogeneous magnetic field is then applied perpendicular to both layers, such that the magnetic flux lines are expelled from the superconductor due to the Meissner effect, and redirected through the superconductor holes coinciding with those of the 2DES. It should be pointed out that the properties of superconductors have been successfully employed to generate spatially varying magnetic fields on 2DES. ${ }^{24-26}$ Likewise, advances in lithographic techniques allow today the fabrication of large area, periodic, and uniform nanohole arrays perforated in different materials. ${ }^{27-29}$

The setup proposed yields a 2DES subject to spatial (equivalent to strong electric) and magnetic modulations of the same period, with the particularity that the magnetic field does not influence directly (classically) the electron motion. For simplicity, in the modelization of such a system we assume a constant value of the magnetic field within the flux tube. However, it is worth mentioning that the calculated band structures only depend on the net flux threading the holes, and not on the particular profile of the magnetic field within the tubes provided it keeps locally the axial symmetry. Therefore, although the experimentally generated flux tubes depart from the constant field picture (as happens in the case of superconductors), the expected results are equivalent to those reported here, as long as the (possible) leakage of magnetic tails into the semiconductor is negligible. 


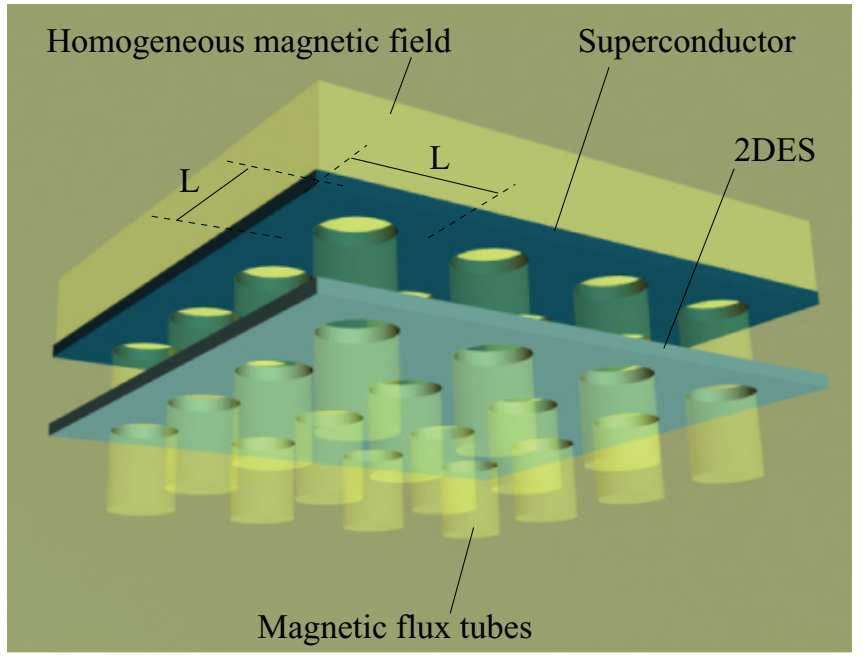

FIG. 1. (Color online) Schematics of the studied system. A square array of nanoholes of period $L$ is patterned simultaneously on both a high-mobility 2DES and a superconducting layer grown on top of it. The regular arrangement of nanoholes, which act as artificial potential pillars (antidots) for the $2 \mathrm{D}$ electrons, yields a periodic, strong potential modulation $V(\mathbf{r})$ in the 2DES layer. A magnetic modulation consisting of magnetic flux tubes piercing the 2DES holes can be obtained by applying a perpendicular magnetic field on top of the whole system. With this setup, the field cannot pierce the superconducting layer (and thus the 2DES), but only the patterned holes.

In the following, we show that the low-lying region of the field-dependent energy spectrum exhibits a Hofstadter-type structure, which is repeated unmodulatedly in periods of one flux quantum. Deviations from Hofstadter's one-band spectrum arise only in the energy axis, due to the coupling between the different Bloch bands. Whereas the square lattice spectrum shows a high resemblance with the well-known butterfly structure, the rectangular lattice presents a quasicontinuous subband structure for lattices with relatively large holes, reflecting the disappearance of Aharanov-Bohm-type quantum interference effects. ${ }^{30}$

\section{THEORETICAL OUTLINE}

The corresponding Hamiltonian for an electron in the highmobility 2DES can be written in atomic units as

$$
H=\frac{1}{2 m}[\mathbf{p}+\mathbf{A}(\mathbf{r})]^{2}+V(\mathbf{r}) .
$$

In Eq. (1), $m$ is the electron effective mass, and $V$ is the periodic modulation defining the hole lattice. Since the holes (of radius $a$ and forming a square pattern of period $L$ ) constitute forbidden regions for the electrons, we set $V=\infty$ within a hole and $V=0$ elsewhere. $\mathbf{A}$ is the potential vector defining the magnetic flux tubes piercing the holes (see Fig. 1). It can be built as the superposition of the individual potential vectors $\mathbf{A}_{n, m}(n, m=0, \pm 1, \pm 2, \ldots)$ describing the magnetic field $\mathbf{B}=(0,0, B)$ through each of the infinite holes of the system:

$$
\mathbf{A}=\sum \mathbf{A}_{n, m},
$$

where $n, m$ label the hole centered in $(x, y, z)=(n L, m L, 0)$ and

$$
\mathbf{A}_{n, m}= \begin{cases}\frac{B}{2}\left(-y^{\prime}, x^{\prime}, 0\right) & \text { if }\left(x^{\prime 2}+y^{\prime 2}\right)^{1 / 2} \leqslant a \\ \frac{B a^{2}}{2}\left(-\frac{y^{\prime}}{x^{\prime 2}+y^{\prime 2}}, \frac{x^{\prime}}{x^{\prime 2}+y^{\prime 2}}, 0\right) & \text { if }\left(x^{\prime 2}+y^{\prime 2}\right)^{1 / 2}>a,\end{cases}
$$

where $\left(x^{\prime}, y^{\prime}, 0\right)=(x-n L, y-m L, 0)$ are the coordinates relative to the center of the hole considered, and $B a^{2} / 2=\Phi / \Phi_{0}$ is the number of flux quanta through each hole.

The eigenvalue equation of the Hamiltonian (1) is solved numerically using the finite difference method in a twodimensional grid mapped in a finite region of the $x y$ plane. Concerning boundary conditions, it should be pointed out that, despite $V(x, y)$ being periodic, pure translations in the $x y$ plane do not commute with the Hamiltonian when $B \neq 0$, as they do not commute with the kinetic energy operator. As shown by Brown, ${ }^{31}$ in the case of a homogeneous magnetic field (defined by a potential vector that we refer to as $\mathbf{A}_{h}$ ) it is possible to define another type of transformation, called magnetotranslations, which do commute with the corresponding Hamiltonian [Eq. (1) with $\mathbf{A}=\mathbf{A}_{h}$ ] and can be used to impose proper boundary conditions in a finite region of the $x y$ plane. Such transformations are defined by the operators:

$$
T_{m}(\mathbf{R})=e^{-i \mathbf{R}\left[\mathbf{p}-\mathbf{A}_{h}(\mathbf{r})\right]},
$$

where $\mathbf{R}$ is a lattice vector and $\mathbf{A}_{h}$ is chosen in the symmetric gauge.

However, in our case $\left(\mathbf{A} \neq \mathbf{A}_{h}\right)$ the operators $T_{m}(\mathbf{R})=$ $e^{-i \mathbf{R}(\mathbf{p}-\mathbf{A}(\mathbf{r}))}$ do not commute with $H$. To overcome this inconvenience let us write $\mathbf{A}$ [Eq. (2)] as follows:

$$
\mathbf{A}=\left(\mathbf{A}-\mathbf{A}_{h}\right)+\mathbf{A}_{h},
$$

where $\mathbf{A}_{h}$ describes a homogeneous magnetic field $\mathbf{B}_{h}$ generating the same flux as $\mathbf{A}\left(\Phi / \Phi_{0}=B_{h} L^{2} / 2 \pi=B a^{2} / 2\right)$ in the crystallographic unit cell [the $(L \times L)$ unit cell for $B=0$ ]. Then, $\mathbf{A}-\mathbf{A}_{h}$ yields a null net flux in the unit cell, which raises the question of whether the potential vector $\mathbf{A}-\mathbf{A}_{h}$ is periodic. It can be easily proved that a periodic vector potential implies a null magnetic flux in the unit cell, but the opposite is not necessarily true. Yet, we can always select the convenient gauge for $\mathbf{A}$ such that $\mathbf{A}-\mathbf{A}_{h}=\mathbf{A}_{p}$, where $\mathbf{A}_{p}$ is a periodic vector potential $\left[\mathbf{A}_{p}(\mathbf{r}+\mathbf{R})=\mathbf{A}_{p}(\mathbf{r})\right]$. In our case, this holds for $\mathbf{A}$ in the gauge given by Eq. (3) and $\mathbf{A}_{h}$ in the symmetric gauge $\mathbf{A}_{h}=-\frac{1}{2}\left(\mathbf{r} \times \mathbf{B}_{h}\right)$. Under these circumstances ( $\mathbf{A}=\mathbf{A}_{p}+\mathbf{A}_{h}$ ), it is straightforward to verify that the operators given by (4) commute with the Hamiltonian (1), and then can be employed to impose the two periodic boundary conditions (in $x$ and $y$ directions) needed to integrate the eigenvalue equation of $H$.

However, one must take into account that the set of magnetic translation operators in two dimensions $\left\{T_{m}(\mathbf{R})\right\}$ form a ray group, which means that in general $\left[T_{m}\left(\mathbf{R}_{1}\right), T_{m}\left(\mathbf{R}_{2}\right)\right] \neq 0$. In particular,

$$
\left[T_{m}\left(\mathbf{R}_{1}\right), T_{m}\left(\mathbf{R}_{2}\right)\right]=2 i \sin \left(\pi \frac{\Phi_{\mathbf{R}_{1}, \mathbf{R}_{2}}}{\Phi_{0}}\right) T_{m}\left(\mathbf{R}_{1}+\mathbf{R}_{2}\right),
$$



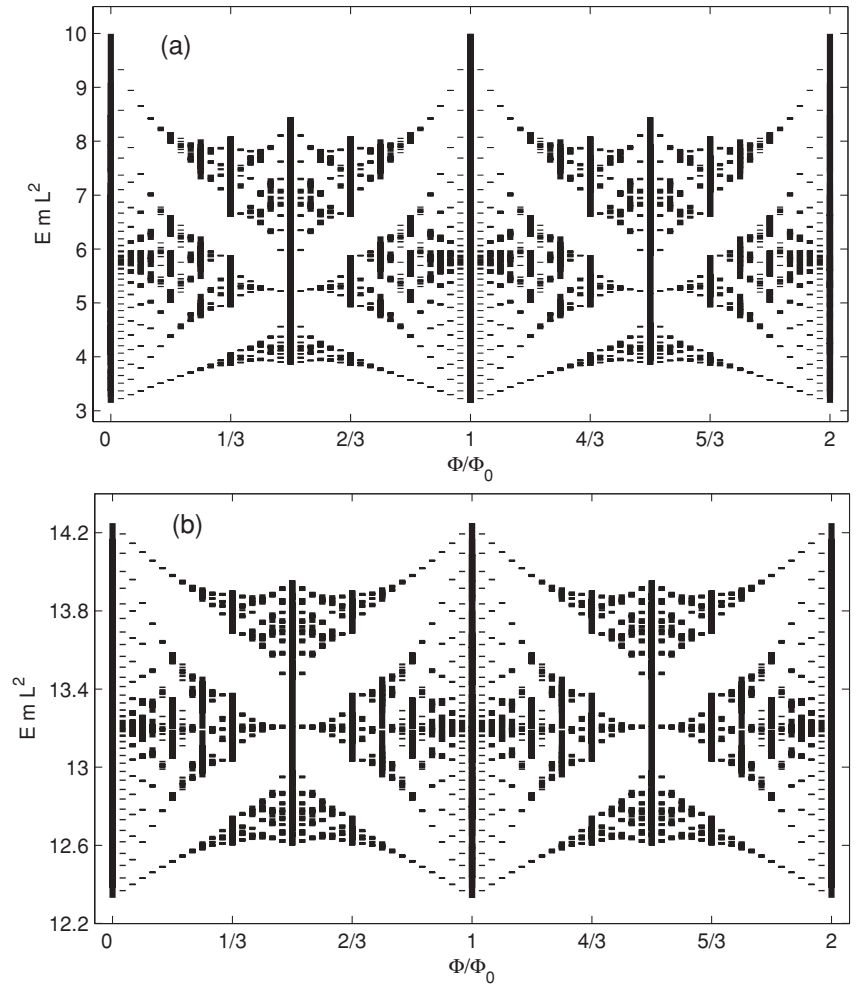

FIG. 2. Energy structure of the square lattice lowest Bloch band as a function of the magnetic flux through a crystallographic unit cell. Results are plotted for two different antidot filling fractions (fraction of area occupied by the holes): (a) $f=0.05$; (b) $f=0.3$. Note the different scale of the energy axis in (a) and (b).

where $\frac{\Phi_{\mathbf{R}_{1}, \mathbf{R}_{2}}}{\Phi_{0}}$ is the magnetic flux quanta piercing the area comprised by $\mathbf{R}_{1} \times \mathbf{R}_{2}$. Thus, only when $\frac{\Phi_{\mathbf{R}_{1}, \mathbf{R}_{2}}}{\Phi_{0}}$ takes integer values the commutator is zero, the group $\left\{n_{1} T_{m}\left(\mathbf{R}_{1}\right), n_{2} T_{m}\left(\mathbf{R}_{2}\right)\right\}$ (with $n_{1}, n_{2} \in Z$ ) is isomorphic to the group of pure inplane translations, and the independently derived boundary conditions in the $x$ and $y$ directions can be safely applied. When the number of flux quanta in the crystallographic unit cell $\left(\Phi / \Phi_{0}\right)$ is not integer, the system experiences a reduction of symmetry, such that only certain subsets of (simultaneously applied) $x$ and $y$ magnetic translations leave the electron state of motion unaltered. These comprise the magnetotranslations defined by new unit cells (magnetic unit cells) pierced by an integer number of flux quanta and compatible in turn with the crystallographic periodicity. In our case, and unless otherwise indicated, we select a square magnetic unit cell comprising 36 crystallographic unit cells, which allows us to obtain the electronic energy structure for the rational flux values $\Phi / \Phi_{0}=n / 36, n$ being an integer.

\section{RESULTS}

Figure 2 shows the lowest Bloch band of the square lattice as a function of the magnetic flux quanta through a single hole. As can be seen, a pattern of minibands and minigaps arise for noninterger values of $\Phi / \Phi_{0}$, with an energy width that is exactly repeated in periods of one flux quantum. For $\Phi / \Phi_{0}=p / q$ with $p, q$ integers and relative prime the band is split up into $q$ nonoverlapping subbands, as can be confirmed
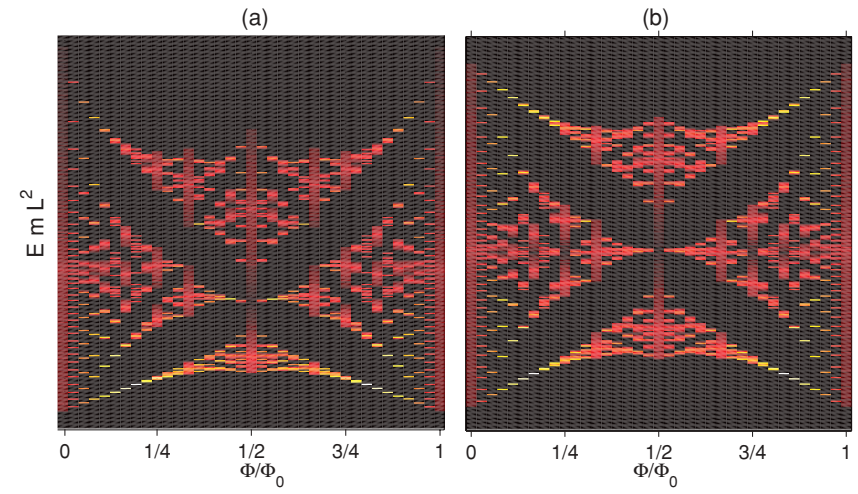

FIG. 3. (Color online) Density of states calculated for the cases displayed in Fig. 2. (a) $f=0.05$; (b) $f=0.3$. Lighter colors indicate larger density of states.

by inspection of the corresponding density of states shown in Fig. 3. ${ }^{32}$ This is the same as holds in the Hofstadter's tight-binding spectrum, though it should be mentioned that this property is not generally fulfilled when band coupling is considered in typically modulated 2DES. Indeed, calculations of antidot superlattices under low homogeneous magnetic fields revealed a partial overlap of the minibands for flux numbers with small denominator. ${ }^{4}$

Figure 2 also reveals that, as a difference with the one-band tight-binding spectrum, previously closed gaps open in our case due to the coupling with excited Bloch bands. This is apparent through the comparison of Figs. 2(a) and 2(b), which differ in the antidot filling fraction $\left(f=\frac{\pi a^{2}}{L^{2}}=0.05\right.$ and 0.3 , respectively). In Fig 2(b), the coupling between the first and more excited Bloch bands is weak, and the spectrum deviates to a lesser extent from Hofstadter's. The symmetry in the energy axis is nearly maintained, and the $q / 2$ th minigap for $\Phi / \Phi_{0}=p / q$ with even $q$ (i.e., the central minigap) remains closed as it holds in the one-band tight-binding spectrum. Instead, for $f=0.05$ (small holes) the coupling becomes noticeable, and the spectrum deviates from this high symmetry picture. Upper subbands become wider than the lower ones, and the closed gaps open for $p / q$ with $q$ even and $q \geqslant 4$. The opening of closed gaps in the Hofstadter spectrum due to the coupling with excited bands has already been reported for electrically modulated 2DES in the quantum Hall regime, having significant implications in the Hall conductance. ${ }^{3}$ However, the opening of the central gap in the latter case is also produced for $p / q=1 / 2$, which might then be related to the direct and nonclassical influence of the magnetic flux on the electron motion. ${ }^{33}$ This possibility raises the question on whether distortions of similar nature arise or not in the weak homogeneous field regime, since in such a regime the direct influence of the field is expected to be small, and the corresponding subband structures are expected to approach those of Fig. 2. Our calculations on a system similar to that sketched in Fig. 1 but where the magnetic flux tubes are substituted by a homogeneous magnetic field (not shown) reveal a close similarity of the subband structure to those of Fig. 2 for very small flux numbers, but as the flux increases disagreements start to show up even in the first butterfly, including a substantial opening of the $\Phi / \Phi_{0}=1 / 2$ central 


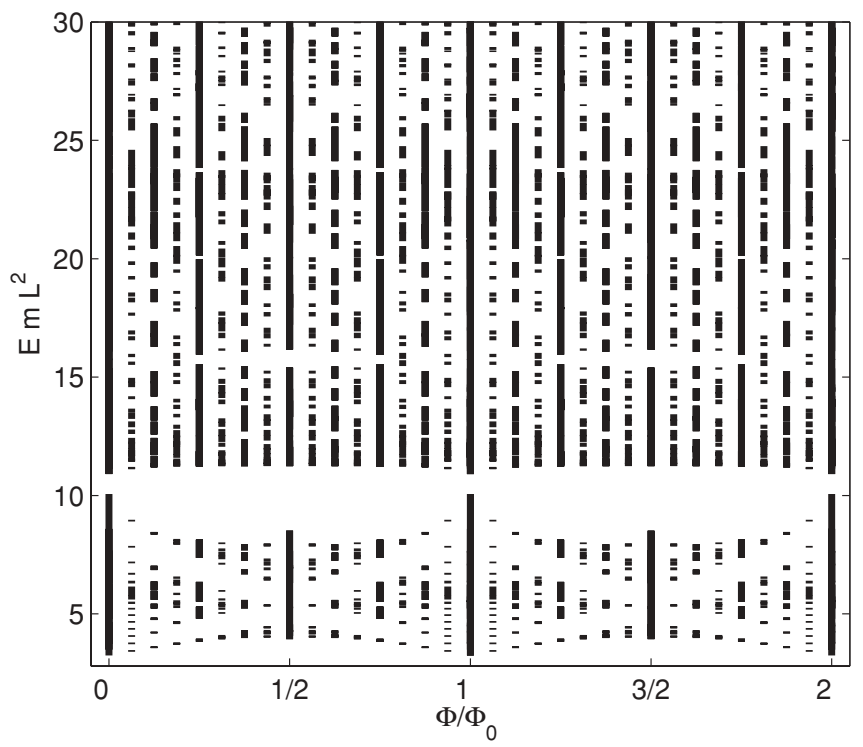

FIG. 4. Lowest-lying energy spectrum for the square lattice with $f=0.05$ as a function of the magnetic flux.

gap. This opening can only be ascribed to the direct influence of the magnetic field on the electron motion, and evidences that this sort of subband distortion is prominent also in the weak homogeneous field regime.

As for the excited bands, the butterfly hides among a complex pattern of overlapping Bloch bands which, obviously, interact strongly. This is shown in Fig. 4 for $f=0.05 .{ }^{34}$ The figure includes the second, third, and fourth Bloch bands, which already overlap in the absence of magnetic field. As can be observed, such overlapping minimizes the number and width of the gaps appearing at rational values of $\Phi / \Phi_{0}$. This would result in an intrinsic difficulty to observe experimentally the subband structure, difficulty that will be increased due to disorder broadening. ${ }^{8-10,35}$

We probe next the magnetic subband structure for the rectangular lattice of period $L \times 2 L$. The magnetic unit cell employed in this case is the same as in Fig. 2, but eliminating alternate rows of holes and the corresponding magnetic flux tubes. This restricts the rational flux numbers studied to $n / 18$. The results are collected in Fig. 5 for the same $a / L$ relations as
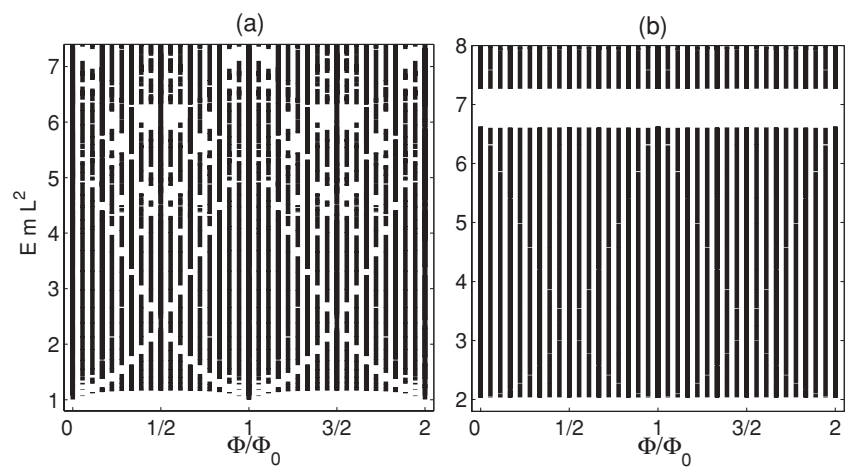

FIG. 5. Lowest-lying energy spectrum for the rectangular lattice with (a) $f=0.05$ and (b) $f=0.3$ as a funtion of the magnetic flux. In the higher energy region of the spectrum represented in (a) the lowest Bloch band overlaps with excited bands. those studied in the square lattice. ${ }^{36}$ For $f=0.3$ (large holes) we observe in Fig. 5(b) an almost complete disappearance of the internal subband structure of the first Bloch band for noninteger flux numbers. Even the band envelope is very slightly modulated as compared with the square lattice case. Instead, for $f=0.05$ [small holes; see Fig. 5(a)], some of the more characteristic gaps still persist, though narrowed by the widened subbands. ${ }^{37}$ This difference with Fig. 5(b) can be explained as coming from the different spatial separation among the holes in the short-period lattice direction, which is larger for the $f=0.05$ than for the $f=0.3$ case. The larger separation opens a channel for field-induced quantum interference phenomena, which is strongly limited for large filling fractions $(f=0.3$ case). This can be better seen in Fig. 6, which shows the corresponding energy structure along the two main lines of the reciprocal lattice related to the electron dispersion in $x$ and $y$. Concerning this figure, it is

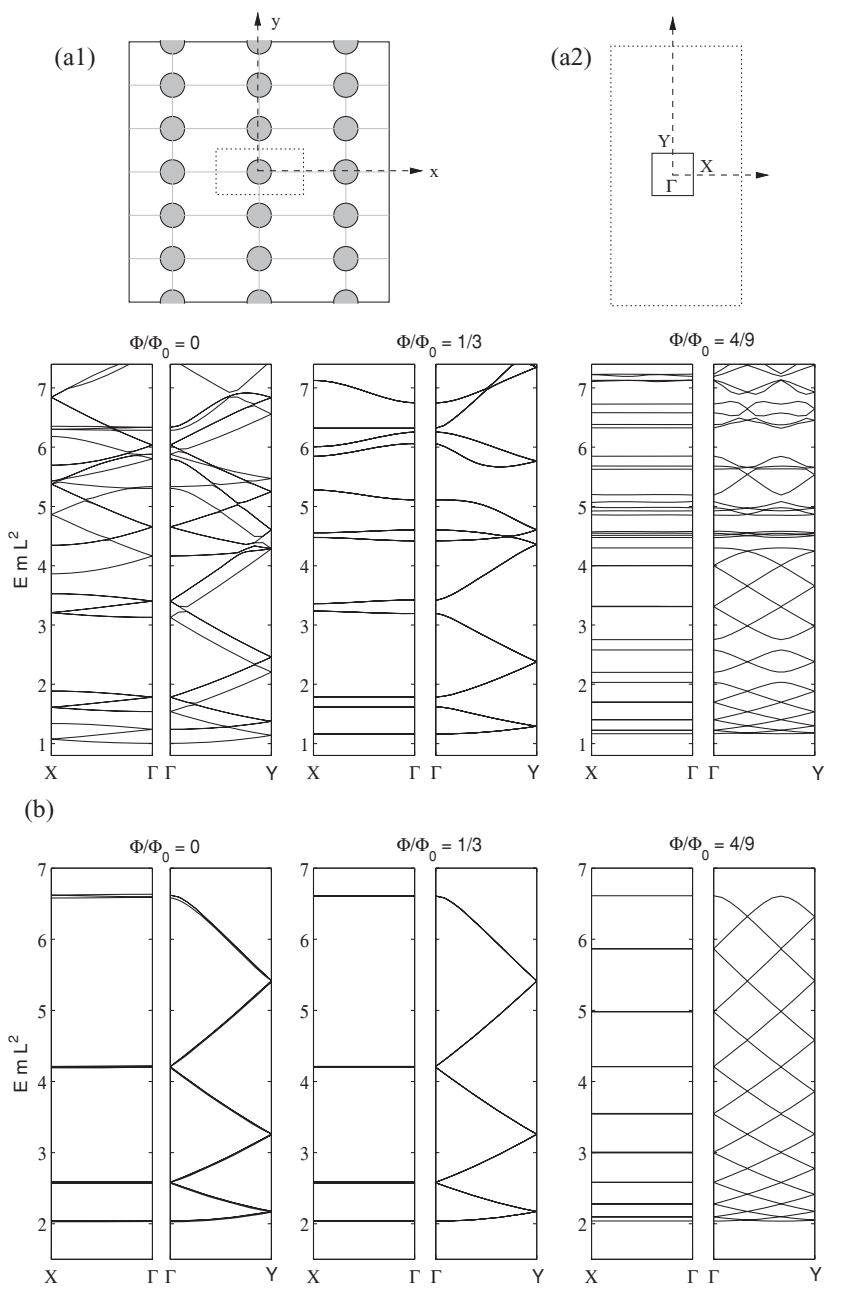

(c)

FIG. 6. Lowest-lying energy structure for the rectangular lattice along the $X-\Gamma$ and $\Gamma-Y$ lines of the two-dimensional reciprocal lattice, for different magnetic fluxes and filling fractions: (b) $f=$ 0.05 ; (c) $f=0.3$. The real and reciprocal lattices are schematized in subfigures (a1) and (a 2), respectively. The dotted lines indicate the limits of the corresponding unit cells at zero field, whereas the solid lines stand for the limits of the cells employed in the calculations (magnetic unit cells at $\Phi / \Phi_{0}=1 / 18$ ). 
(a)
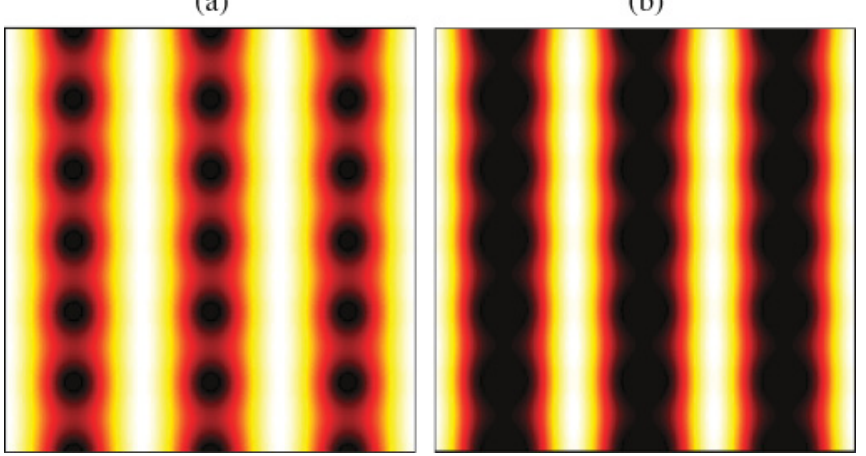

FIG. 7. (Color online) Zero-field ground-state electron density in the magnetic unit cell of the rectangular lattice. (a) $f=0.05$; (b) $f=0.3$. Lighter colors indicate higher electron densities.

worth remembering here that the cell employed in all the calculations enclose 18 crystallographic unit cells. This is the origin of the apparent band bending observed for the represented fluxes (a feature of the calculation method that will occur for any flux $\Phi / \Phi_{0}=p / q$ with $q$ lower than 18). Then, note that labels $X$ and $Y$ in Fig. 6 do not represent the border of the magnetic Brillouin zone, but only label directions in the reciprocal lattice. Thus, $\Gamma-X / \Gamma-Y$ corresponds to the large or short period lattice direction. The figure shows that for $f=0.05$ and $\Phi / \Phi_{0}=0$ the band structure is clearly anisotropic, being less dispersive for the electron propagation in the large-period lattice direction. The interference phenomena induced by the magnetic field flatten the subband structure in this direction, whereas open small gaps in the other one. Conversely, for $f=0.3$ the band structure shows no dispersion in this direction even for $\Phi / \Phi_{0}=0$. This reveals the singly connected character of the corresponding wave functions, which extend only along the wider channels opened among the holes (see Fig. 7). Thus, field-induced interference phenomena are negligible, and the featureless band structure of Fig. 5(b) can be interpreted, regardless of the magnetic flux, in terms of a superposition of noninteracting, highly dispersive one-dimensional (1D) bands along the short-period lattice direction. As a consequence, rectangular lattices are not suitable candidates to provide experimental verification of the Hofstadter-type spectrum, since the limited field dependence of the miniband dispersion is not expected to yield significant changes of the band conductivity contribution to magnetotransport as a function of the magnetic field. ${ }^{4}$

\section{CONCLUDING REMARKS}

We studied the magnetic subband structure of a highmobility 2DES patterned with square and rectangular arrays of holes (antidots) pierced by magnetic flux tubes. Within this scheme, the coupling of the magnetic field with the 2D electrons is purely quantum mechanical. The square lattice exhibits a Hofstadter-type spectrum with an unmodulated envelope. The symmetry of the butterfly is maintained in the field axis, whereas minor deviations, induced by the coupling among Bloch bands, arise in the energy axis. Conversely, field-induced quantum interference phenomena are masked in rectangular lattices. This reduces the number and width of open gaps, and yields an almost featureless spectrum for large antidot filling fractions. As a consequence, significant features in the magnetoresistance coming from field-induced variations of the band conductivity are not expected for rectangular lattices.

As it disentangles classical and purely quantum interference effects, the experimental realization of the system studied could provide further insight into the features observed in the low-field magnetoresistance of electrically modulated two-dimensional electron systems.

\section{ACKNOWLEDGMENTS}

Support from MICINN Project No. CTQ2008-03344, and UJI-Bancaixa Project No. P1-1A2009-03 is acknowledged. *josep.planelles@qfa.uji.es

${ }^{1}$ U. Rössler and M. Suhrke, in Advances in Solid State Physics, edited by B. Kramer (Springer, Berlin, 2000), Vol. 40, pp 35-50.

${ }^{2}$ D. R. Hofstadter, Phys. Rev. B 14, 2239 (1976).

${ }^{3}$ D. Springsguth, R. Ketzmerick, and T. Geisel, Phys. Rev. B 56, 2036 (1997).

${ }^{4}$ P. Rotter, M. Suhrke, and U. Rössler, Phys. Rev. B 54, 4452 (1996).

${ }^{5}$ T. Schösser, K. Ennsslin, J. P. Kotthaus, and M. Holland, Europhys. Lett. 33, 683 (1996).

${ }^{6}$ C. Albrecht, J. H. Smet, K. von Klitzing, D. Weiss, V. Umansky, and H. Schweizer, Phys. Rev. Lett. 86, 147 (2001).

${ }^{7}$ M. C. Geisler, J. H. Smet, V. Umansky, K. von Klitzing, B. Naundorf, R. Ketzmerick, and H. Schweizer, Phys. Rev. Lett. 92, 256801 (2004); Physica E 25, 227 (2004).

${ }^{8}$ M. Koshino and T. Ando, J. Phys. Soc. Jpn. 73, 3243 (2004).

${ }^{9}$ B. Huckestein, Phys. Rev. Lett. 72, 1080 (1994).

${ }^{10}$ K. Yang and R. N. Bhatt, Phys. Rev. B 59, 8144 (1999).
${ }^{11}$ M. Kato, A. Endo, S. Katsumoto, and Y. Iye, Phys. Rev. B 77, 155318 (2008).

${ }^{12}$ D. Weiss, K. Richter, A. Menschig, R. Bergmann, H. Schweizer, K. von Klitzing, and G. Weimann, Phys. Rev. Lett. 70, 4118 (1993).

${ }^{13}$ D. Weiss, M. L. Roukes, A. Menschig, P. Grambow, K. von Klitzing, and G. Weimann, Phys. Rev. Lett. 66, 2790 (1991).

${ }^{14}$ H. Silberbauer and U. Rössler, Phys. Rev. B 50, 11911 (1994).

${ }^{15}$ R. Onderka, M. Suhrke, and U. Rössler, Phys. Rev. B 62, 10918 (2000).

${ }^{16}$ R. Neudert, P. Rotter, U. Rössler, and M. Suhrke, Phys. Rev. B 55, 2242 (1997).

${ }^{17}$ R. Schuster, K. Ensslin, J. P. Kotthaus, M. Holland, and C. Stanley, Phys. Rev. B 47, 6843 (1993).

${ }^{18}$ S. Meckler, T. Heinzel, A. Cavanna, G. Faini, U. Gennser, and D. Mailly, Phys. Rev. B 72, 035319 (2005).

${ }^{19}$ Y. Iye, M. Ueki, A. Endo, and S. Katsumoto, J. Phys. Soc. Jpn. 73, 3370 (2004). 
${ }^{20}$ See, for example, R. R. Gerhardts, D. Weiss, and U. Wulf, Phys. Rev. B 43, 5192 (1991); R. Schuster, K. Ensslin, D. Wharam, S. Kühn, J. P. Kotthaus, G. Böhm, W. Klein, G. Tränkle, and G. Weimann, ibid. 49, 8510 (1994); R. B. S. Oakeshott and A. MacKinnon, J. Phys. Condens. Matter 6, 1519 (1994); D. E. Grant, A. R. Long, and J. H. Davies, Phys. Rev. B 61, 13127 (2000); O. Yevtushenko, G. Lütjering, D. Weiss, and K. Richter, Phys. Rev. Lett. 84, 542 (2000); P. Vasilopoulos, X. F. Wang, and F. M. Peeters, Phys. Rev. B 69, 035331 (2004); M. Khoury, A. M. Lacasta, J. M. Sancho, A. H. Romero, and K. Lindenberg, ibid. 78, 155433 (2008).

${ }^{21}$ X. F. Wang, P. Vasilopoulos, and F. M. Peeters, Phys. Rev. B 70, 155312 (2004).

${ }^{22}$ Another setup avoiding classical effects (a waveguide with controllable scatterers) was used successfully in U. Kuhl and H.-J. Stöckmann, Phys. Rev. Lett. 80, 3232 (1998) to capture aspects of the butterfly structure in the transmission spectra of microwaves.

${ }^{23}$ In order to access the regime of the Hofstadter butterfly energy spectrum in magnetotransport the system must be coupled to the continuous spectrum of the leads, yielding Fano-like resonances and distorting the isolated system spectrum [see, e.g., S. Ujevic and M. Mendoza, Phys. Rev. B 82, 035432 (2010); J. Skjånes, E. H. Hauge, and G. Schön, ibid. 50, 8636 (1994)]. These resonances allow in turn the electron to transit from the system to the lead. Additionally, the experimental observation will also be masked by disorder. Ultimately, it is the property of the recursive structure of the energy spectrum that enables experimental observation of the internal band structure, for only primary gaps separating clusters of subbands will survive in any physical realization. In this sense, the system we propose will be linked to the same limitations as the antidot lattice pierced by a homogenous magnetic field, except for the distortion of Hofstadter-type subband structure by an increasing field. This lack of modulation will favor the experimental observation of the recursive structure of the energy spectrum.

${ }^{24}$ A. K. Geim, I. V. Grigorieva, S. V. Dubonos, J. G. S. Lok, J. C. Maan, A. E. Filippov, and F. M. Peeters, Nature (London) 390, 259 (1997).

${ }^{25}$ A. K. Geim, S. V. Dubonos, J. G. S. Lok, I. V. Grigorieva, J. C. Maan, L. T. Hansen, and P. E. Lindelov, Appl. Phys. Lett. 71, 2379 (1997).

${ }^{26}$ J. Reijniers, F. M. Peeters, and A. Matulis, Phys. Rev. B 59, 2817 (1999).
${ }^{27}$ W. Wu, D. Dey, O. G. Memis, A. Katsnelson, and H. Mohseni, Nanoscale Res. Lett. 3, 123 (2008); W. Wu, D. Dey, A. Katsnelson, O. G. Memis, and H. Mohseni, J. Vac. Sci. Technol. B 26, 1745 (2008).

${ }^{28}$ F. M. Huang, T. S. Kao, V. A. Fedotov, Y. Chen, and N. I. Zheludev, Nano Lett. 8, 2469 (2008).

${ }^{29}$ A. Weidinger, Europhysics News 35, 152 (2004); J. H. Zollondz and A. Weidinger, Nucl. Instrum. Methods B 225, 178 (2004).

${ }^{30}$ Y. Aharonov and D. Bohm, Phys. Rev. 115, 485 (1959).

${ }^{31}$ E. Brown, Phys. Rev. 133, A1038 (1964).

${ }^{32}$ The splitting into $q$ subbands cannot be seen straightforwardly in Fig. 2 due to the existence of closed gaps. For example, at $\Phi / \Phi_{0}=1 / 2$ the two minibands that can be distinguished in Fig. 3 touch each other, and therefore are represented as a single bar in Fig. 2 .

${ }^{33}$ Other deviations from Hofstadter's spectrum are found in Ref. 3, mainly related to band bending and flat band conditions, connected in turn with the field-induced modulation of the band envelope..$^{21}$ These are not present in our case, due to the absence of envelope modulation.

${ }^{34}$ Because of the more demanding calculation, we have employed in this case a magnetic unit cell comprising 16 crystallographic unit cells.

${ }^{35} \mathrm{An}$ additional source of distortion of the subband structure is the presence of defects, such as the lack of one or more holes (antidots) in the lattice. Conversely to the homogeneous field case, in the proposed setup this lack not only disturbs the spatial periodicity, but also the magnetic one. However, we have checked (not shown) that the Hofstadter spectrum obtained is as robust against the presence of defects as it is its homogeneous field counterpart.

${ }^{36}$ The corresponding filling fractions (fraction of area occupied by the holes) are thus halved, since the crystallographic unit cell has been doubled. However, for the sake of a better comparison with the square lattice we keep here the definition of $f=\frac{\pi a^{2}}{L^{2}}$ employed before.

${ }^{37}$ This is in agreement with the trends found by Hasegawa et al. [Y. Hasegawa, Y. Hatsugai, M. Kohmoto, and G. Montambaux, Phys. Rev. B 41, 9174 (1990)] as a consequence of the introduction of anisotropy in the square lattice. 